\title{
Transatlantica
}

Revue d'études américaines. American Studies Journal

\section{The Birth of a Nation de Nate Parker, une anti-} adaptation?

\section{Michaël Roy}

\section{OpenEdition}

\section{Journals}

Édition électronique

URL : https://journals.openedition.org/transatlantica/11625

DOI : $10.4000 /$ transatlantica. 11625

ISSN : 1765-2766

Éditeur

Association française d'Etudes Américaines (AFEA)

Référence électronique

Michaël Roy, « The Birth of a Nation de Nate Parker, une anti-adaptation ? », Transatlantica [En ligne], 1 I 2018, mis en ligne le 05 septembre 2019, consulté le 02 février 2023. URL : http://

journals.openedition.org/transatlantica/11625; DOI : https://doi.org/10.4000/transatlantica. 11625

Ce document a été généré automatiquement le 2 février 2023.

\section{(c) $(1)$}

Creative Commons - Attribution - Pas d'Utilisation Commerciale - Pas de Modification 4.0 International - CC BY-NC-ND 4.0

https://creativecommons.org/licenses/by-nc-nd/4.0/ 


\title{
The Birth of a Nation de Nate Parker, une anti-adaptation?
}

\author{
Michaël Roy
}

1 Sur le bandeau d'une traduction française de The Confessions of Nat Turner (Confessions de Nat Turner) publiée en janvier 2017 par les éditions Allia, on lit ceci : "Le récit à l'origine du film The Birth of a Nation de Nate Parker $»^{1}$. L'affirmation, qui semble faire du film de Nate Parker une adaptation des Confessions, est problématique à plus d'un titre.

2 Confessions de Nat Turner, rappelons-le, est une brochure parue à Baltimore en 1831, peu après la révolte de Nat Turner en Virginie. Long d'une vingtaine de pages dans son édition originale, le texte constitue une source d'information essentielle sur le déroulement de la révolte ainsi que sur la personnalité et les motivations de son instigateur : l'esclave rebelle y livre sa version des faits, à la première personne, dans ce qui s'apparente moins à un "récit d'esclave " (slave narrative) à la Frederick Douglass qu'à une « confession de criminel » (criminal confession); le genre a aux États-Unis une longue histoire, au sein de laquelle les Africains-Américains tiennent une place importante (pour ne pas dire prépondérante), au point qu'on a pu faire de la «littérature de potence» (gallows literature) le lieu d'émergence de la voix autobiographique noire (DeLombard). L'existence même des Confessions explique - en partie du moins - la place centrale qu'occupe Nat Turner dans l'histoire et la mémoire américaines par rapport à d'autres figures d'esclaves révoltés moins connues du grand public, tels Gabriel Prosser, Charles Deslondes et Denmark Vesey. Confessions de Nat Turner est toutefois le produit de circonstances particulières : il ne s'agit pas d'un récit écrit par Turner lui-même, mais de la retranscription d'une série d'entretiens menés à l'initiative d'un certain Thomas R. Gray, avocat blanc de Virginie, alors que Turner est en prison et qu'il attend son jugement. Issu d'une famille de propriétaires d'esclaves, Gray est tout sauf un intermédiaire neutre, comme le révèle sa préface aux Confessions, où les rebelles apparaissent en fanatiques assoiffés de sang :

No cry for mercy penetrated their flinty bosoms. No acts of remembered kindness made the least impression upon these remorseless murderers. Men, women and children, from hoary age to helpless infancy were involved in the same cruel fate. 
Never did a band of savages do their work of death more unsparingly. (Confessions of Nat Turner 4)

C'est peu de dire que Gray oriente la lecture du témoignage de Nat Turner. Il y va de son intérêt personnel, puisque Gray est alors lourdement endetté : il espère tirer profit de cet opuscule, qu'on ne manquera pas de s'arracher dès lors que le caractère sensationnel en est parfaitement établi (Breen, 2015 146-147). On comprend pourquoi la critique s'est longtemps interrogée sur la fiabilité du document: faut-il croire Gray lorsqu'il affirme dans la préface avoir fidèlement rapporté les propos de Turner - «I determined [...] to commit his statements to writing, and publish them, with little or no variation, from his own words " (Confessions of Nat Turner 3-4) - ou bien considérer que la voix de l'esclave se trouve déformée dès lors que c'est un "Sudiste raciste blanc » $(a$ white Southern racist) qui la relaie (Fabricant 335) ? Patrick Breen, auteur du dernier ouvrage en date sur la révolte (2015), conclut à partir d'une analyse de la structure des Confessions que celles-ci préservent malgré tout la voix et le point de vue de Nat Turner.

3 Nate Parker entretient pour sa part un rapport ambigu aux Confessions. Dans le dossier de presse de son film de 2016, The Birth of a Nation, il reconnaît s'être familiarisé avec le texte, tout en précisant l'avoir approché avec précaution : «There are many aspects of the supposed confessions that have come under fire. There were no witnesses to the confession and some of the things said seem to be completely out of line with who Turner was by common knowledge ». Le propos est vague, mais il traduit une évidente méfiance à l'endroit des Confessions en général et de Thomas Gray en particulier : « He had every motivation to fabricate the things that were said", ajoute Parker en interview (Yamato). De fait, Parker ne prétend pas adapter les Confessions, et sans doute verrait-il d'un mauvais œil qu'on en fasse le récit « à l'origine » de The Birth of a Nation. Il gomme délibérément la présence intrusive de l'avocat blanc, qui ne figure ni en ouverture ni dans la brève scène d'emprisonnement qui précède l'exécution. L'ensemble du film peut s'interpréter comme une tentative de " rendre sa voix » à Nat Turner (Laurent), ici débarrassée de tout filtre - celui imposé par Thomas Gray, mais aussi celui imposé par William Styron près d'un siècle et demi plus tard. On se rappelle la polémique suscitée par son roman Les Confessions de Nat Turner (la reprise du titre est éloquente) à sa sortie en 1967 : qu'un riche Blanc de Virginie, un WASP dont la grandmère avait elle-même possédé des esclaves, s'empare d'une figure noire mythique et parle en son nom ne pouvait qu'entraîner la colère d'une partie au moins des intellectuels africains-américains, et ce, en plein mouvement pour les droits civiques. Comme les auteurs de William Styron's Nat Turner: Ten Black Writers Respond (1968) avant lui, Nate Parker s'est montré extrêmement critique à l'égard des Confessions de Styron : "Styron's book was a work of fiction; a falsified re-imagining he used to propagate his own misguided and paternalistic ideas of Nat Turner and his motives » (Rezayazdi). Le réalisateur se présente en dépositaire légitime de la parole de Turner, contre ceux qui l'ont usurpée à des fins racistes ou mercenaires.

4 S'il se débarrasse du dispositif dialogique des Confessions de 1831 - dialogique au sens où deux voix s'y font entendre entre la préface et le corps du texte, mais aussi parce que Gray interrompt ponctuellement Turner pour lui poser des questions -, Parker conserve, pour ne pas dire amplifie, un aspect essentiel du document original, à savoir la focalisation sur la figure de Turner. On aurait pu imaginer, pour rompre nettement avec les représentations passées, un traitement choral de la révolte, qui fasse une véritable place à Hark, Will, Nelson et aux autres esclaves mentionnés par Turner. Ce n'est pas le choix opéré par Nate Parker, loin s'en faut : alors même que le titre du film 
annonçait la naissance d'une nation, c'est-à-dire d'une communauté, Parker s'emploie à construire un héros, c'est-à-dire un être singulier, sur lequel toute l'attention du cinéaste est concentrée. La critique a souligné les limites esthétiques et politiques de cette héroïsation exacerbée du personnage : dans un article intitulé «Why We Don't Need Another Hero (Film): Nate Parker's The Birth of a Nation", Kenneth Warren regrette que Parker n'ait pas pris davantage de libertés par rapport aux conventions formelles du biopic hollywoodien; pour l'historienne Vanessa Holden, c'est la dimension collective de la révolte qui est perdue de vue : «By crafting a narrative that depicts a hero made in his own image, Parker fails to explore [...] the vast resistive network of "heroes" needed to pull off a violent slave revolt ». Des Confessions de Nat Turner à The Birth of a Nation, le point de vue adopté reste celui de Nat Turner, source de fascination (tantôt morbide, tantôt admirative) pour Thomas Gray comme pour Nate Parker.

5 Mais le parallèle entre le scripteur et le réalisateur s'arrête là car, pour l'essentiel, Parker prend ses distances avec les Confessions. L'un des enjeux de The Birth of a Nation est en effet de rendre la révolte de Nat Turner intelligible pour le spectateur ordinaire, qu'un tel épisode de violence pourrait choquer. Pour que le massacre final paraisse acceptable, tout ce qui a précédé doit y conduire de manière inéluctable. Or le témoignage de Turner ne se prête pas aisément à une telle lecture. Si Parker se détache des Confessions, c'est avant tout parce que ce texte ne lui permet pas de raconter l'histoire qu'il souhaite raconter.

6 D'un point de vue narratif, l'accent est mis dans le film sur l'horreur de la condition d'esclave, avec des images parfois difficilement soutenables. La scène où l'on retire ses dents à un esclave à coups de burin afin de pouvoir le nourrir de force est l'une des plus violentes de tout le corpus cinématographique sur l'esclavage : «[it] is so brutal that it is impossible to convey its power ", écrit une journaliste (Page-Kirby). La scène de punition au fouet après un acte de désobéissance de la part de Nat Turner n'est pas moins éprouvante pour le spectateur, figuré à l'écran par la foule des esclaves obligés d'assister à ce spectacle qui doit leur servir de leçon; la mère (Aunjanue Ellis) et la grand-mère (Esther Scott) de Nat finissent par détourner le regard lorsque les coups gagnent en intensité. Loin de toute stylisation, Nate Parker fait le choix de montrer, en gros plan si besoin, les corps meurtris des esclaves - la cervelle éclatée d'un fugitif, le visage tuméfié de Cherry Turner (Aja Naomi King), le dos lacéré de Nat. Afin de susciter ces scènes de violence, Parker invente une tournée pendant laquelle Nat Turner aurait eu à prêcher l'obéissance aux esclaves du comté de Southampton : ce dispositif permet de montrer comment, d'une plantation à l'autre, l'esclave ouvre les yeux sur ce qui l'entoure et découvre que la relation de proximité qu'il entretient avec son maître Samuel Turner (Armie Hammer) tient de l'exception plutôt que de la règle. Ainsi la violence de la révolte à la fin de The Birth of a Nation est-elle à la mesure de celle exercée à l'encontre des esclaves tout au long du film. C'est là un point de divergence entre The Birth of a Nation et les Confessions, où l'enchainement des causes et des effets n'est pas aussi évident. Non seulement Turner n'évoque jamais la violence de l'esclavage dans son témoignage, mais il affirme qu'à la veille de la révolte, il appartenait à un maître qui le traitait bien: "Since the commencement of 1830, I had been living with Mr. Joseph Travis, who was to me a kind master, and placed the greatest confidence in me; in fact, I had no cause to complain of his treatment to me " (Confessions of Nat Turner 11). Y a-t-il eu censure de la part de Gray, qui aurait effacé toute référence explicite à la sombre réalité de l'esclavage et usé du stéréotype du bon maître pour mieux mettre en 
avant - à l'inverse de Parker - la sauvagerie des esclaves ? Cela parait peu plausible, dans la mesure où les Confessions ont justement pour but de révéler les motifs qui ont poussé Nat Turner à se rebeller : "You have asked me to give a history of the motives which induced me to undertake the late insurrection ", dit Turner à Gray en ouverture de son récit (Confessions of Nat Turner 7). Turner ne fait pas de tel ou tel traitement cruel l'élément déclencheur de l'insurrection, ce qui laisse penser que celle-ci est motivée par un refus systémique de l'esclavage comme institution immorale et injuste plutôt que par un désir de vengeance personnelle. C'est ce qu'ont noté plusieurs historiennes et historiens, à l'instar de Patrick Breen - «For Turner, [...] slavery as it was provided ample justification for his revolt. A slave need not have experienced the most heinous crimes to reject this system » (Breen, 2016) - et de Leslie Alexander :

By all accounts, Turner took up arms against slavery because he believed slavery was morally wrong and violated the law of God. [...] This fact is important because it demonstrates that black people not only fought against slavery because of its extreme violence and brutality, but also because they knew in their hearts that slavery was an unjust, exploitative system that violated moral laws. In other words, they fought simply because they wanted to be free. (Alexander)

7 La dimension religieuse de l'acte de révolte occupe une large place dans les Confessions et elle constitue en définitive sa seule justification: Nat Turner se décrit comme un prophète dont les visions divines l'ont convaincu qu'il avait pour mission de mener une révolte d'esclaves. Nate Parker ne reprend que partiellement ces éléments (les visions sont à peine suggérées dans The Birth of a Nation) et rationnalise le schéma qui pousse Turner à l'action en injectant dans son film des scènes de violence nombreuses et répétées. C'est, plus exactement, le viol de sa femme Cherry qui décide Nat à se révolter, ce qui a valu à Parker des critiques d'autant plus vives qu'a refait surface au moment de la sortie du film une affaire de viol dans laquelle le réalisateur avait été impliqué. Selon Leslie Alexander et Salamishah Tillet, Parker se sert de ses personnages féminins - Esther (Gabrielle Union), la femme de Hark (Colman Domingo), est également victime d'un viol - pour mieux célébrer l'héroïsme viril des protagonistes masculins, défenseurs de femmes silencieuses et impuissantes. Rien de tel ne figure dans les Confessions, même si le viol des esclaves noires par leur maître blanc était une pratique courante. 


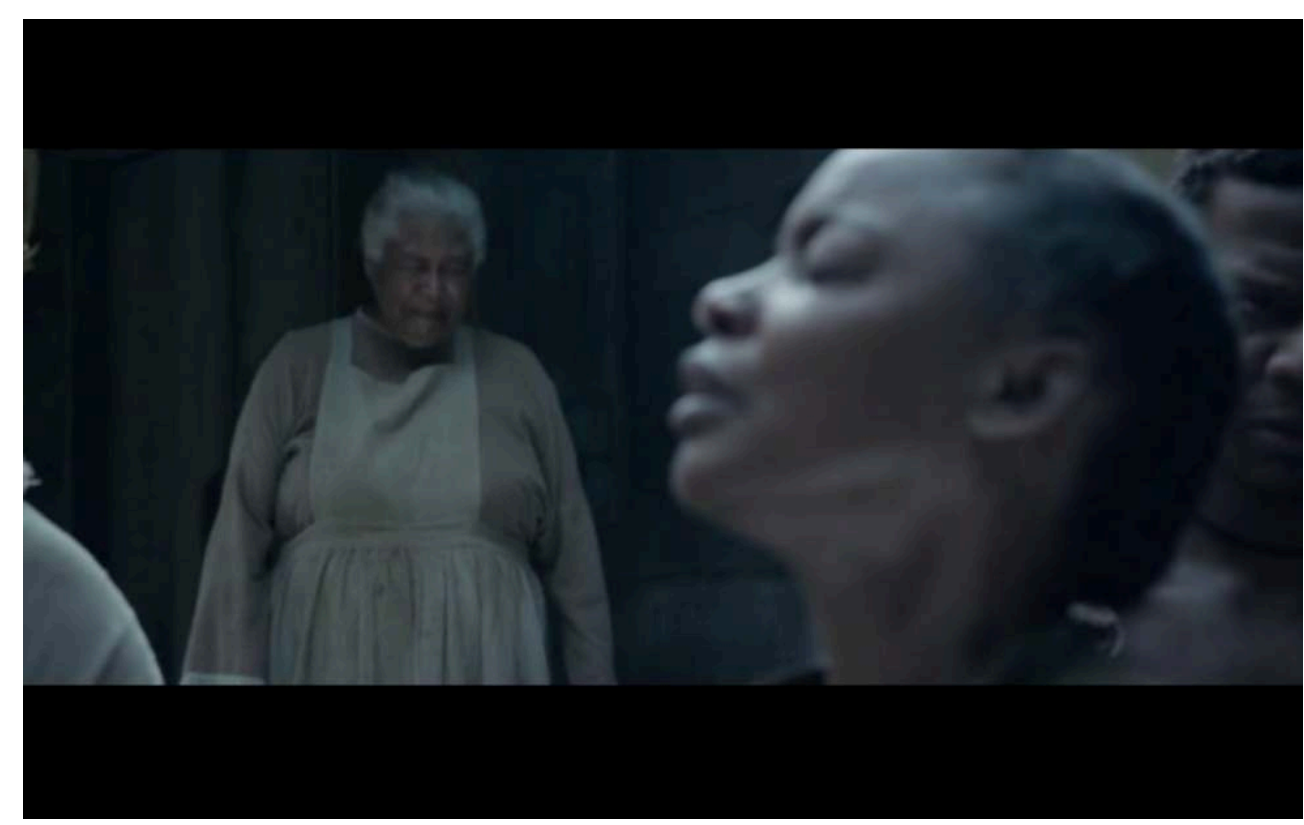

C'est donc un Nat Turner bien différent que donnent à voir les Confessions et The Birth of a Nation. Tout est fait dans le film pour amener la spectatrice et le spectateur à éprouver de l'empathie pour le personnage. Nat Turner y est digne, généreux, respecté des autres esclaves ; c'est un bon père de famille, touché par l'amour de sa femme et de sa fille. L'injustice et la violence auxquelles il est confronté n'en paraissent que plus intolérables. L'image que Nat Turner donne de lui-même dans les Confessions - à supposer qu'elle n'ait pas été façonnée par Thomas Gray - est plus complexe. En tant que prophète, Turner considère qu'il est différent de ses pairs. Il se tient en marge de la communauté des esclaves et cultive volontairement le mystère: "Having soon discovered to be great, I must appear so, and therefore studiously avoided mixing in society, and wrapped myself in mystery, devoting my time to fasting and prayer » (Confessions of Nat Turner 8-9). Il suscite à l'occasion l'incompréhension des autres esclaves, par exemple lorsqu'il fuit la plantation où il est retenu pour finalement $\mathrm{y}$ revenir au prétexte que «l'Esprit » lui aurait dit de «servir son maître ici-bas » ( the Spirit appeared to me and said [...] that I should return to the service of my earthly master »); il cite à ce propos un passage de la Bible (Luc 12:47) que l'on trouve plus souvent sous la plume des esclavagistes : «For he who knoweth his Master's will, and doeth it not, shall be beaten with many stripes " (Confessions of Nat Turner 9-10). Enfin décidé à lancer la révolte, il hésite quant à la meilleure façon de mener les opérations, tergiverse au point de s'en rendre malade et de retarder le déclenchement de l'insurrection. À l'opposé du Nat Turner de The Birth of a Nation, figure courageuse qu'une trajectoire clairement définie mène de l'obéissance à la résistance via un certain nombre d'épreuves touchant les siens et lui-même, le Nat Turner des Confessions est un personnage trouble, contradictoire et parfois indécis. Lors de la révolte, il est notable que Nat Turner ne parvienne en fin de compte à faire qu'une seule victime, la jeune Margaret Whitehead ; à plusieurs reprises, ses coups de hache ou d'épée ratent, et c'est Will qui doit achever ses victimes pour lui. Nate Parker montre quant à lui un Nat Turner déterminé surgissant de l'ombre pour porter un coup sec et fatal à son maître (fig. 2) $)^{2}$. 


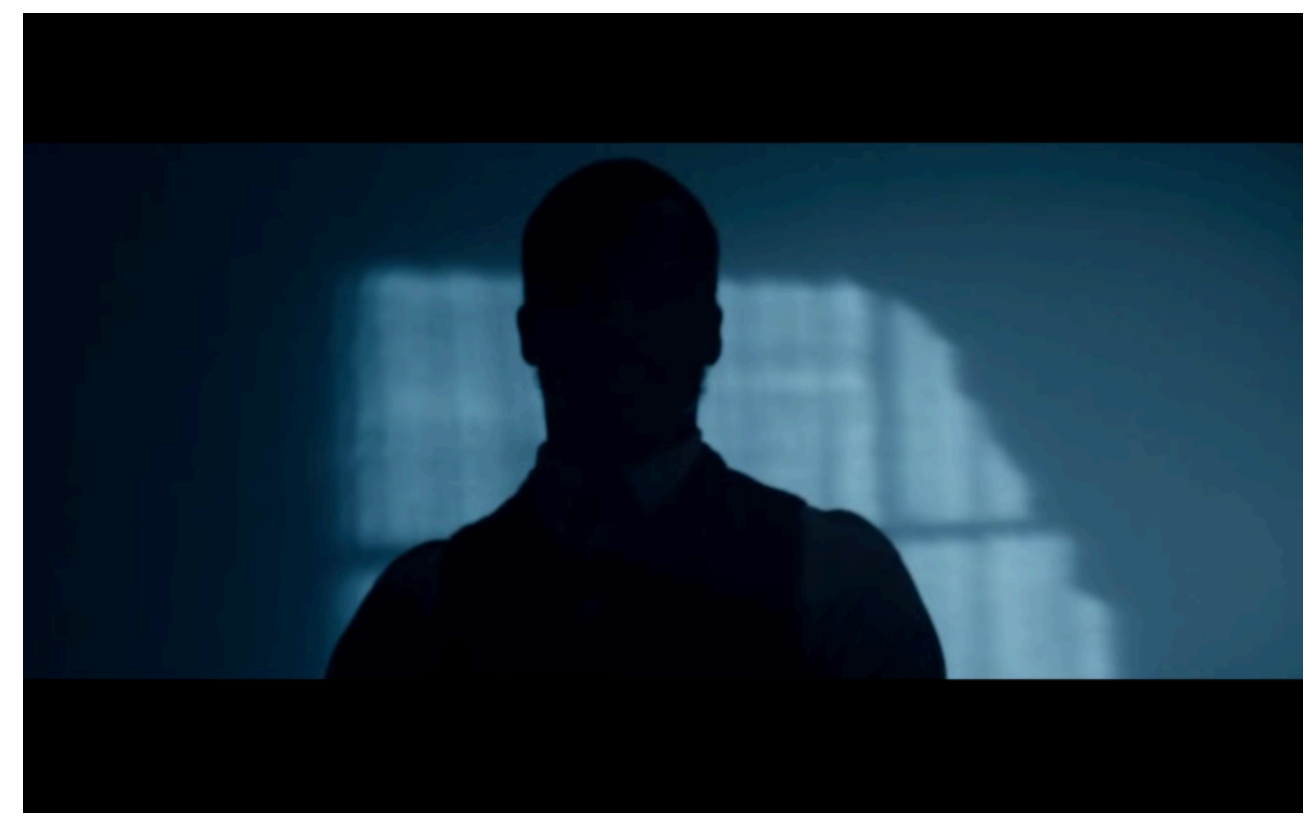

Ce coup, le spectateur l'entend mais ne le voit pas. Alors que Parker a révélé toute l'horreur des sévices imposés aux esclaves et qu'il reprend ce thème à la fin du film lorsqu'est évoqué le mouvement de représailles dont sont victimes des Noirs innocents, la révolte en tant que telle est traitée sur un mode allusif, en une quinzaine de minutes, sur un film de près de deux heures. Parker n'en montre que certains aspects et en écarte d'autres évoqués dans les Confessions. Parce qu'il ne consacre qu'un temps limité à l'épisode insurrectionnel, le cinéaste renonce de fait à représenter le caractère systématique de la révolte, qui voit les rebelles se rendre de maison en maison et tuer les Blancs qui s'y trouvent, femmes et enfants compris; dans un passage mémorable des Confessions, deux insurgés reviennent sur leurs pas et tuent un nourrisson qu'ils avaient oublié. La radicalité de la révolte vient entre autres de ce qu'elle n'épargne personne. Seuls des hommes semblent trouver la mort, dans The Birth of a Nation, et tous ont commis des actes répréhensibles : Samuel Turner, devenu alcoolique, a fait fouetter Nat et a rendu possible le viol d'Esther ; Jethro (Justin M. Smith), lorsqu'il est tué par Will (Chiké Okonkwo), est au lit avec une très jeune esclave; Raymond Cobb (Jackie Earle Haley), tué par Nat d'un coup de poignard dans la gorge lors de la confrontation armée à Jerusalem, a participé au viol de Cherry. La logique du « il pour œil, dent pour dent » est poussée à son comble dans la scène où Will demande à décapiter luimême un maître dont il dit plus tard avoir eu à subir les coups de fouet. Il ne s'agit plus, comme dans les Confessions, d'un implacable déchaînement de violence visant à supprimer tout individu blanc ayant quelque lien que ce soit avec un système inique, mais d'une juste punition infligée à ceux qui y sont le plus directement impliqués ${ }^{3}$.

Par bien des aspects, The Birth of a Nation se démarque des Confessions, comme pour mieux dire le peu de crédit que Nate Parker accorde au texte retranscrit par Thomas Gray, dont le film est en quelque sorte une "anti-adaptation ». La tonalité étrange, mystique, parfois hallucinée du témoignage cède la place à un récit plus conventionnel, mais paradoxalement présenté par Nate Parker comme plus fidèle à l'esprit de la révolte. Surtout, The Birth of a Nation entend parler de la révolte de Nat Turner sur un mode qui puisse saisir l'attention du grand public, susciter son adhésion et le faire réfléchir - aux dires du cinéaste - à la persistance de l'oppression raciale dans l'Amérique contemporaine. Parker cherche à faire œuvre de pédagogie à travers son 
film, ce qui implique pour lui de s'émanciper du texte qui a justement permis à Nat Turner de passer à la postérité.

\section{BIBLIOGRAPHIE}

ALEXANDER, Leslie M. « The Birth of a Nation Is an Epic Fail ». The Nation, 6 octobre 2016, https:// www.thenation.com/article/the-birth-of-a-nation-is-an-epic-fail/. Page consultée le 15 novembre 2018.

«Birth of a Nation (The): A Roundtable ». Civil War History, vol. 64, n 1, 2018, p. 56-91.

BREEN, Patrick H. « Birth of a Nation, The Historian's Review: A Scholar Considers Use of The Past in Parker's Movie-For-Today ». Deadline, 7 octobre 2016, https://deadline.com/2016/10/birth-ofa-nation-historians-review-use-of-past-1201832168/. Page consultée le 15 novembre 2018.

BREEN, Patrick H. The Land Shall Be Deluged in Blood: A New History of the Nat Turner Revolt. New York : Oxford University Press, 2015.

CLARKE, John Henrick, dir. William Styron's Nat Turner: Ten Black Writers Respond. Boston : Beacon Press, 1968.

Confessions de Nat Turner. Éd. et trad. Michaël Roy. Paris : Allia, 2017.

Confessions of Nat Turner (The), the Leader of the Late Insurrection in Southampton, Va. Baltimore :

Thomas R. Gray, 1831.

DELOMBARD, Jeannine Marie. In the Shadow of the Gallows: Race, Crime, and American Civic Identity. Philadelphie : University of Pennsylvania Press, 2014.

FABRICANT, Daniel S. " Thomas R. Gray and William Styron: Finally, a Critical Look at the 1831 Confessions of Nat Turner ». American Journal of Legal History, vol. 37, n 3, 1993, p. 332-361.

HOLDEN, Vanessa. « The Trouble in Nate Parker's Southampton: The Birth of a Nation, a Review ». Process: A Blog for American History, 6 octobre 2016, http://www.processhistory.org/holden-birthof-a-nation/. Page consultée le 15 novembre 2018.

LAURENT, Sylvie. " "The Birth of a Nation met en cause la mémoire nationale" des États-Unis ». Mediapart, 14 janvier 2017, https://www.mediapart.fr/journal/culture-idees/140117/birthnation-met-en-cause-la-memoire-nationale-des-etats-unis?onglet=full. Page consultée le 15 novembre 2018.

PAGE-KIRBY, Kristen. « The Birth of a Nation Is Hard to Watch. Watch Anyway ». Washington Post, 7 octobre 2016, https://www.washingtonpost.com/express/wp/2016/10/07/the-birth-of-a-nationis-hard-to-watch-watch-anyway/?noredirect=on\&utm_term=.1282cfd4974c. Page consultée le 15 novembre 2018.

REZAYAZDI, Soheil. « Five Questions with The Birth of a Nation Director Nate Parker ». Filmmaker Magazine, 25 janvier 2016, https://filmmakermagazine.com/97103-five-questions-with-the-birthof-a-nation-director-nate-parker/\#.WztiUKmkKRt. Page consultée le 15 novembre 2018. 
TILLET, Salamishah. « How The Birth of a Nation Silences Black Women ». New York Times, 12 octobre 2016, https://www.nytimes.com/2016/10/16/movies/how-the-birth-of-a-nationsilences-black-women.html. Page consultée le 15 novembre 2018.

YAMATO, Jen. « The Birth of a Nation: Meet Nate Parker, the Revolutionary Filmmaker Behind the Sundance Smash ». Daily Beast, 28 janvier 2016, https://www.thedailybeast.com/the-birth-of-anation-meet-nate-parker-the-revolutionary-filmmaker-behind-the-sundance-smash. Page consultée le 15 novembre 2018.

WARREN, Kenneth W. « Why We Don't Need Another Hero (Film): Nate Parker's The Birth of a Nation ». Los Angeles Review of Books, 4 novembre 2016, https://www.lareviewofbooks.org/article/ why-we-dont-need-another-hero-film-nate-parkers-the-birth-of-a-nation. Page consultée le 15 novembre 2018.

\section{NOTES}

1. Je suis l'auteur de la traduction, mais le bandeau promotionnel est de la responsabilité de l'éditeur.

2. Après avoir tué Samuel Turner, Nat Turner est certes pris de vomissements, ce qui traduit peu ou prou les hésitations et faiblesses du Turner des Confessions, en même temps que cela humanise le personnage.

3. Pour une analyse similaire, voir « Birth of a Nation » 60 .

\section{INDEX}

Thèmes : Perspectives

\section{AUTEUR}

\section{MICHAËL ROY}

Université Paris Nanterre, CREA, michael.roy@parisnanterre.fr 